\title{
Study on deformation of surrounding rock in fault tunnel based on field measured data statistics
}

\author{
Chen $\mathrm{Hao}^{1, \mathrm{a}^{*}}$ \\ ${ }^{1}$ School of Highway, Chang'an University, Xi'an, PR China
}

\begin{abstract}
Based on the statistical analysis of surrounding rock deformation from 56 fault tunnels in China, the overall distribution characteristics and time evolution laws of surrounding rock deformation and the relationships with buried depth of tunnel and the height-span ratio are studied. The results of the statistical analysis show that the deformation is excessive when the tunnel passes through the fault zone. The average deformation value of surrounding rock in railway fault tunnels is maximum and second in highway fault tunnels and minimum in subway fault tunnels. The average value of peripheral convergence is larger than that of vault settlement. The deformation increases with the increases of buried depth, and the deformation in railway fault tunnels shows obvious concentration with the change of the height-span ratio. There is a significant time evolution law of surrounding rock deformation in fault tunnels, which can be roughly divided into three stages: rapid growth, slow growth and stabilization. The results offer some references for the optimal design of supporting structure and the reasonable construction time of secondary lining when the tunnels pass through the fault zone.
\end{abstract}

\section{Introduction}

In order to meet the needs of the rapid development of transportation in China, the building of highway and railway tunnels have been continuously developing to a scale of "longer, larger and deeper". Fault fracture zone is the most common adverse geological condition in the construction of tunnel, and tunnel construction will inevitably cross the fault fracture zone during the construction. The design and construction of tunnel crossing fault fracture zone has always been a big problem during the tunnel construction. To date, lots of researches have been conducted and abundant achievements have been made in the construction technologies and mechanical characteristics of the tunnel crossing the fault zone by scholars at home and abroad. For example, Kimura et al. ${ }^{[1]}$ described the flexible and rigid supporting structures used in the construction of Enasan No.2 tunnel through two large fault compression zones, and then compared and analyzed the tunnel displacement under different support structures. Based on the model tests and numerical simulation methods, Jeon et al. ${ }^{[2]}$ studied the influence of faults and weak planes on the stability of tunnels, and believed that the shear displacement of the weak surface would cause significant displacement of the tunnel surrounding rock. Kun et al. ${ }^{[3]}$ studied the influence of the fault zone on metro tunnel in Turkey by combing field monitoring, indoor tests and numerical simulations, and aimed to evaluate the original support strength of the tunnel and determine the risk area when the shallow buried section of the tunnel passing through the fault zone. Wang et al. ${ }^{[4]}$ used the finite element software to simulate the dynamic excavation process of tunnel crossing the fault zone, and analyzed the influence of fault dip angle and width on deformation and pressure of tunnel surrounding rock.

When the tunnel passes through the fault zone, the geological causes and the geological conditions are complex as well as the role between tunnel surrounding rock and supporting structure. So it is very important to monitor and measure the tunnel construction in fault zone. On the one hand, the monitoring and measurement of fault tunnel can timely grasp the deformation and pressure states of surrounding rock and supporting structures in fault zone, which can be used to guide the construction. On the other hand, it can accumulate the experiences and data to provide an analogy basis for design and construction of similar fault tunnel in the future. In recent years, with the increasing number of fault tunnel constructions in China, a large number of measured data have been accumulated. Many scholars have collected a large amount of tunnel monitoring data and analyzed them with statistical methods, and found that the deformation and pressure of the tunnel surrounding rock have obvious regularity and predictability in the time and space distribution. In terms of deformation, Liu et al. ${ }^{[5]}$ made statistics on monitoring data of vault subsidence and surrounding convergence of dozens of domestic expressway tunnels constructed by NATM. The laws of surrounding rock deformation stability time, stability

* Corresponding author: ${ }^{\text {a }}$ chenhao920816@163.com 
value and tunnel surrounding rock grade after initial support construction were summarized and obtained, and the stability evaluation indexes of tunnel surrounding rock deformation under various surrounding rock grades were provided. Zhao et al. ${ }^{[6]}$ conducted statistics on the deformation of 11 large-section loess tunnels on the Zhengxi Passenger Dedicated Line, and quantitatively studied on the deformation laws and reserved deformations of large-section loess tunnels with grade IV and V surrounding rock. Based on the vault settlement and horizontal convergence data of 103 mountain tunnels in China, Fang et al. ${ }^{[7]}$ analyzed the relationship between the deformation value, the stability time and the grade of the surrounding rock, tunnel excavation area, and buried depth.

It could be found that a large number of scholars have monitored the deformation during fault tunnel construction and have accumulated a wealth of measured data at this stage. However, the research on collecting, summarizing and analyzing these data of fault tunnel is relatively rare. Based on the measured data of 134 sections of 56 domestic tunnels crossing fault zones, this paper analyzes the overall distribution characteristics and time regularity of surrounding rock deformation when tunnels pass through fault fracture zone. And then the relationships between surrounding rock deformation and tunnel buried depth, excavation span are studied, in order to provide theoretical support for the design and construction scheme of tunnel in fault fracture zone.

\section{Statistical samples of tunnel site monitoring}

\subsection{Statistical samples}

In this paper, a total of 134 monitoring sections of 56 fault tunnels were statistically analyzed, including 23 railway tunnels, 30 highway tunnels and 3 urban subway tunnels. It should be noted that the statistical samples date is all from published academic papers.

\subsection{Overall distribution characteristics of surrounding rock deformation of fault tunnels}

Fig. 1 and Fig. 2 show the overall distribution characteristics of vault settlement and horizontal convergence of fault tunnels. It can be seen from Fig. 1(a) and Fig. 2(a) that the vault settlement and surrounding convergence of fault tunnels account for the largest proportion in the range of $0-50 \mathrm{~mm}$, both of which are greater than $50 \%$. It indicates that more than half of fault tunnels are well controlled during construction. With the increases of deformation interval, the proportion of section monitoring data decreases significantly. The 50$100 \mathrm{~mm}$ interval and the $150-200 \mathrm{~mm}$ interval account for relatively high proportions, both exceeding $10 \%$. The proportion of surrounding rock deformation exceeding $300 \mathrm{~mm}$ accounts for $6.4 \%$ and $7.3 \%$. At this time, the surrounding rock of the tunnel is already in a large deformation. If the corresponding measures are not taken in time, it is likely to cause disasters such as tunnel collapse. The proportion of vault settlement data in the low value area $(0-100 \mathrm{~mm})$ is $72.7 \%$, which is greater than the surrounding convergence $(66.7 \%)$, indicating that the lateral deformation is greater than the vertical deformation in the fault tunnels.

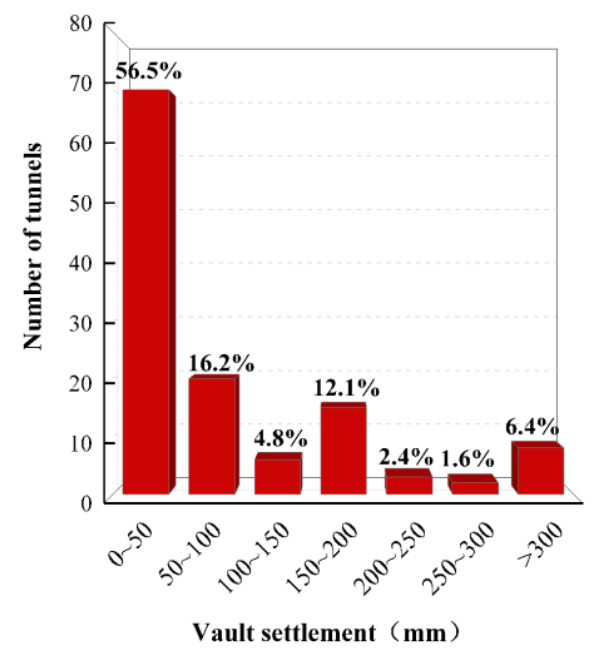

(a)

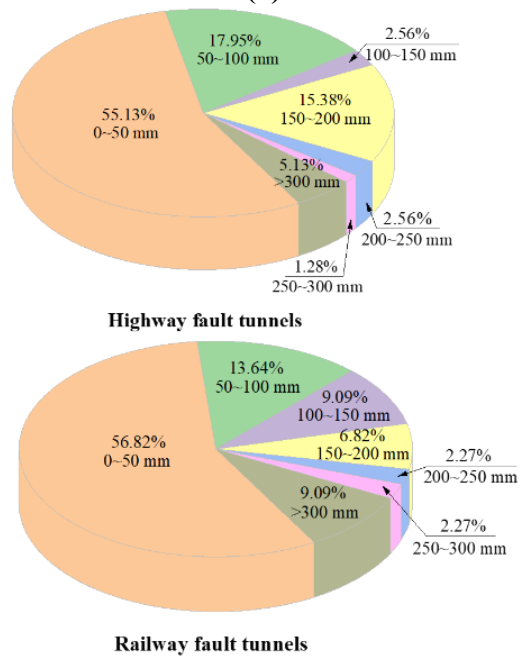

(b)

Fig. 1 Distribution characteristics of vault settlement of fault tunnels

It can be seen from Fig. 1(b) and Fig. 2(b) that the distribution of the surrounding rock deformation of highway fault tunnels is similar to that of railway fault tunnels, and both account for about $50 \%$ in the range of 0 $50 \mathrm{~mm}$. The difference is that the surrounding rock deformation of highway fault tunnels accounts for a large proportion in the range of $150-200 \mathrm{~mm}$, while that of railway fault tunnels is larger in the range of $100-150 \mathrm{~mm}$. When the railway tunnels pass through the fault zones, the surrounding rock convergence proportion in the low value area $(0-100 \mathrm{~mm})$ is smaller than that of the highway tunnels, which indicates that the lateral deformation in the railway fault tunnel is relatively larger. The surrounding rock deformation values of subway fault tunnels are all concentrated in the range of $0-50 \mathrm{~mm}$, which means that the displacement of subway tunnel is well controlled when crossing the fault zones. This is also related to the fact that there are fewer samples of fault section displacement of subway tunnels collected. 


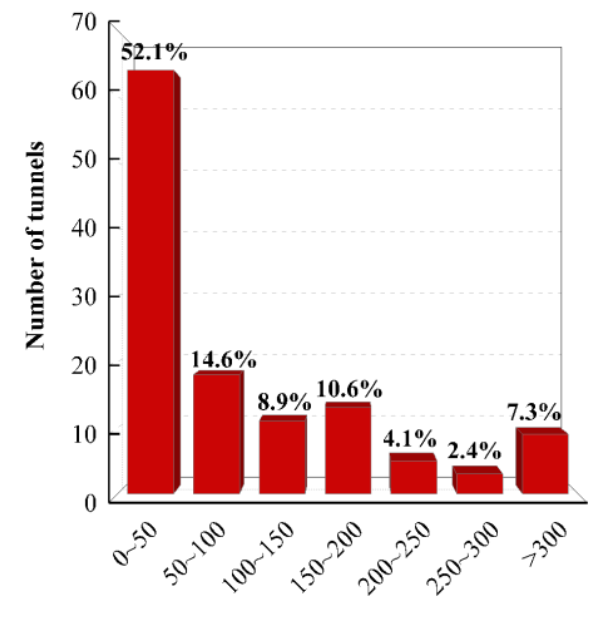

(a)

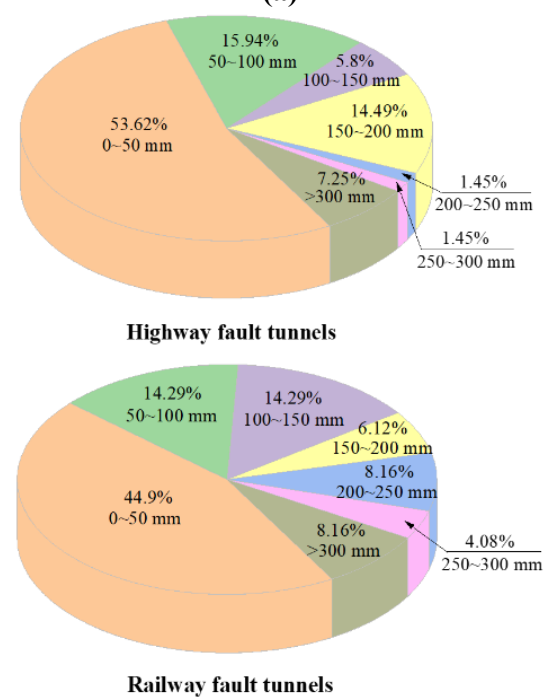

(b)

Fig. 2 Distribution characteristics of convergence of fault tunnels

Table 1 shows the statistical average values of surrounding rock deformation of various types of fault tunnels. The statistical average values of vault settlement and surrounding convergence in fault tunnels are 103.57 $\mathrm{mm}$ and $115.18 \mathrm{~mm}$, which are far greater than the average deformation values of grade $\mathrm{V}$ surrounding rock $(41.0 \mathrm{~mm}$ and $22.2 \mathrm{~mm}$ ) in mountain tunnels calculated by Fang Qian ${ }^{[7]}$, approaching or exceeding the warning value (In "Technical Rules for Highway Tunnel Construction" [8], the reserved deformation in tunnel design should be considered as the ultimate displacement during tunnel construction. If the tunnel displacement measurement result exceeds $2 / 3$ of the ultimate displacement, the corresponding measures must be taken to control the surrounding rock deformation.). Compared with the ordinary grade $\mathrm{V}$ surrounding rock, the geological conditions of the tunnel site are more complex and the surrounding rocks are more broken due to the existence of faults. The surrounding rock deformation caused by the tunnels crossing the fault zones is more significant. From the deformation of fault tunnel surrounding rock, the deformation in railway tunnel is the largest, followed by highway tunnel and the subway tunnel is lowest; the average value of convergence is greater than the average value of vault settlement. The reason for the large deformation of the surrounding rock in railway fault tunnels may be that the buried depth of the railway tunnels is large, and most of the fault belts that pass through are large faults or active faults with large widths and complex geostress conditions. Due to the high construction technologies and strict control standards, the shield method or shallow burying method adopted by the subway tunnels, which can effectively control the deformation, so that the deformation can be controlled in a small range even when passing through fault zones or other adverse geological conditions.

\section{Analysis of influencing factors}

\subsection{Influence of the buried depth}

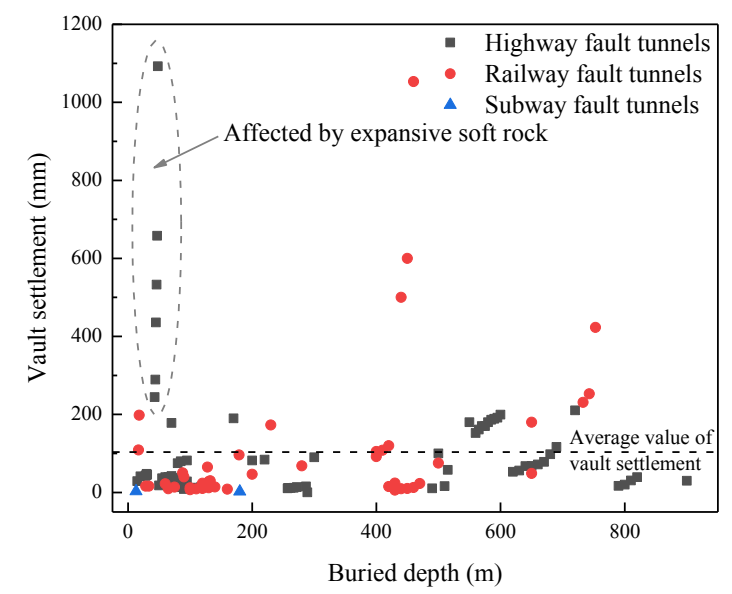

Fig. 3 Relationships between vault settlement of surrounding rock and buried depth in fault tunnels

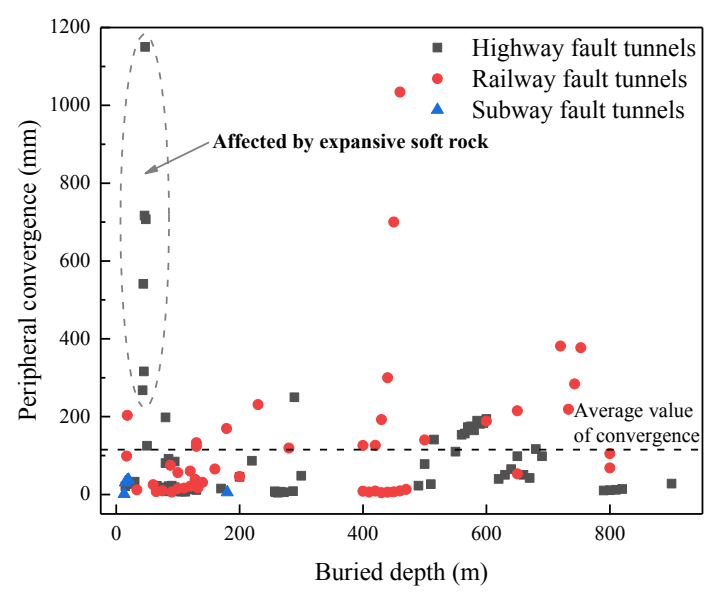

Fig. 4 Relationships between convergence of surrounding rock and buried depth in fault tunnels

Fig. 3-4 show the relationship between surrounding rock deformation and tunnel buried depth of fault tunnels. According to the analysis in the Fig. 3-4, the distribution of deformation data of fault tunnels with buried depth is relatively discrete. However, except for the data in shallow buried section affected by expansive soft rock, the deformation in fault tunnels has an increasing trend with 
the increases of tunnel buried depth. In the range of less than $200 \mathrm{~m}$ buried depth, most of the deformation data are below the average value. As the buried depth increases, more and more deformation data appear above the average value. In the case of more than $400 \mathrm{~m}$, the data of large deformation increases significantly. This feature appears in both highway and railway fault tunnels. In addition, it can be found that compared with the highway fault tunnels, the deformation of the surrounding rock in the railway fault tunnels is more easily affected by the tunnel buried depth.

\subsection{Influence of the height-span ratio}

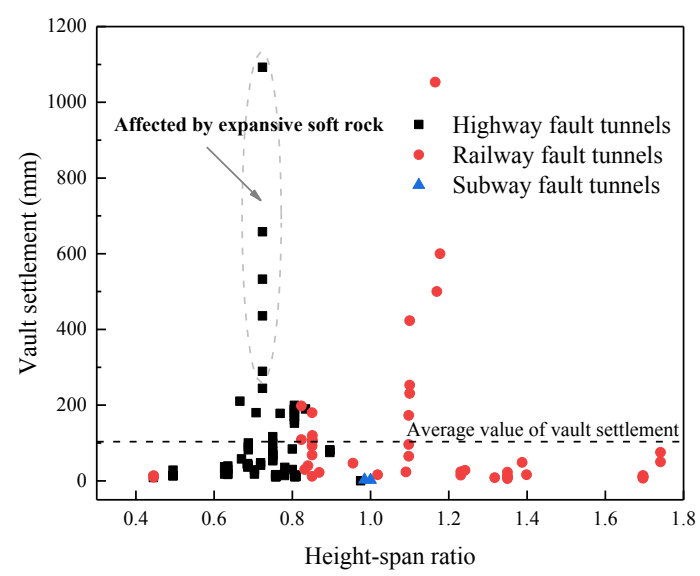

Fig. 5 Relationships between vault settlement of surrounding rock and height-span ratio in fault tunnels

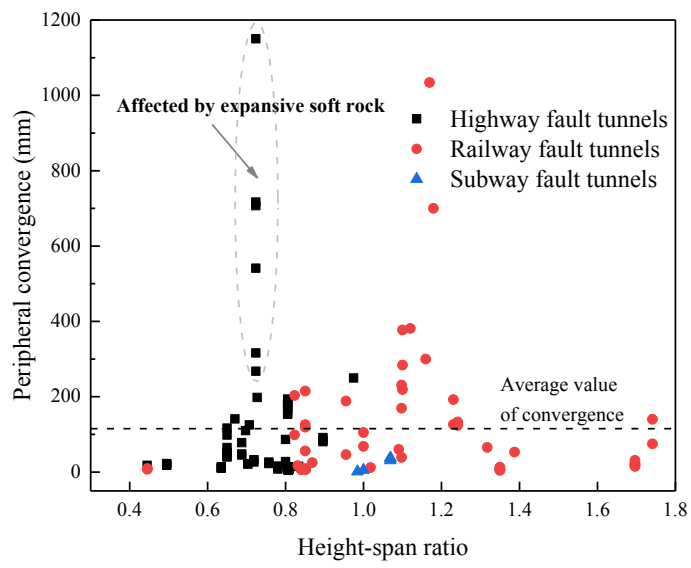

Fig. 6 Relationships between convergence of surrounding rock and height-span ratio in fault tunnels

Fig. 5-6 show the relationship between vault settlement/convergence of surrounding rocks and heightspan ratio of tunnels. In terms of vault settlement, except for the tunnels affected by expansive soft rock, the larger values of vault settlement in highway fault tunnels are mainly concentrated in the range of $0.6-0.8$ of height-span ratio, showing a certain concentration. The larger values of vault settlement in railway fault tunnels are concentrated in the range of $0.8-0.85$ and 1.1-1.2, showing obvious concentration. In terms of surrounding rock convergence, the correlation between convergence in highway fault tunnels and height-span ratio is small, while shows obvious concentration in railway fault tunnels, which is also concentrated in the range of $0.8-0.85$ and 1.1-1.2. It is not found that there is any correlation between the deformation data and the height-span ratio in subway fault tunnels, due to the lack of deformation data.

\section{Temporal characteristics of surrounding rock deformation}

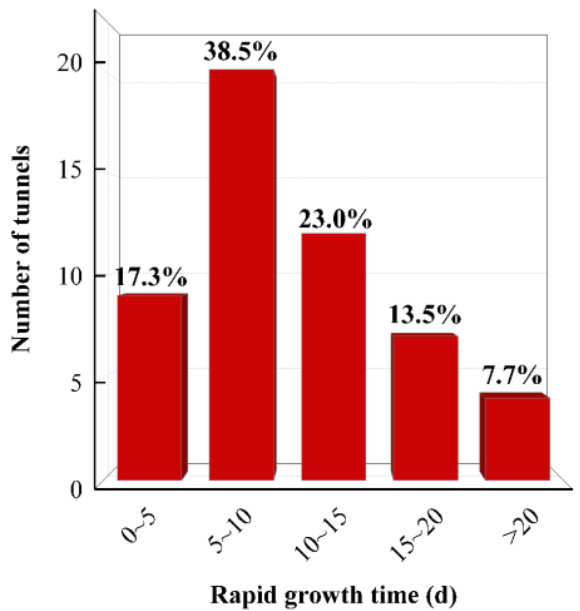

(a) Vault settlement

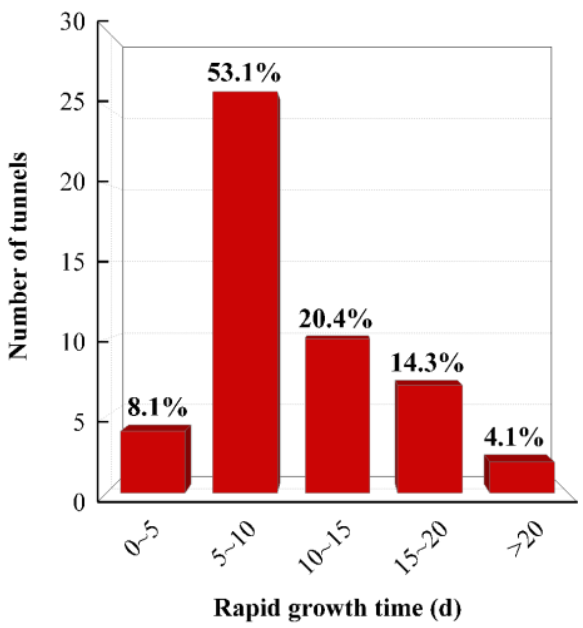

(b) Peripheral convergence

Fig. 7 The rapid growth stage of surrounding rock deformation of fault tunnels

According to the statistical curve analysis of the deformation of surrounding rocks with time, $72.2 \%$ of highway fault tunnel sections and $74.3 \%$ of railway fault tunnel sections vault settlement can be divided into three stages: rapid growth, slow growth and stabilization. The sections with convergence in accordance with this characteristics accounted for $70.6 \%$ and $69.4 \%$ of the highway fault tunnels and railway fault tunnels, respectively. The time effect of surrounding rock deformation of most fault tunnels is significant after excavation.

Fig. 7 shows the statistical analysis of the rapid growth stage of the surrounding rock deformation in fault tunnels. It can be found that the rapid growth time of surrounding rock deformation in fault tunnels is mainly concentrated in 5-10 days, and nearly $80 \%$ of the deformation ends in 15 days. The average time of rapid growth of vault 
settlement and convergence is 9.69 days and 8.73 days, respectively. In the rapid growth stage, the vault settlement and convergence value account for $64.2 \%$ and $65.6 \%$ of the total displacement, respectively. It means that the surrounding rock in the fault tunnels could produce significant deformation immediately after excavation, and the deformation time lasts for a long time. Therefore, it is necessary to strengthen the support in time after the fault tunnels excavation in order to shorten the time of rapid growth stage of deformation, and make deformation enter the slow growth or stable stage as soon as possible.

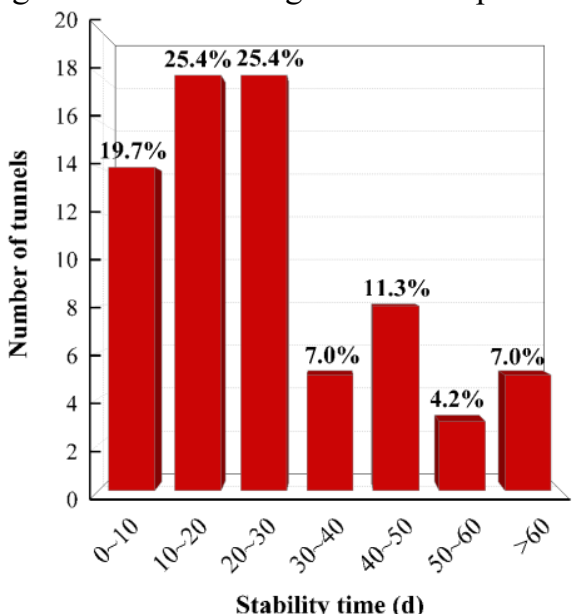

(a)

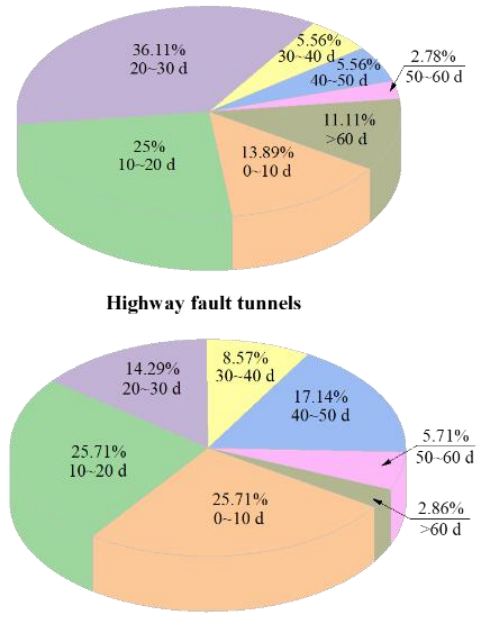

Railway fault tunnels

(b)

Fig. 8 The stability time of vault settlement of fault tunnels

Fig. 8-9 show the stability time distribution of surrounding rock deformation in fault tunnels. It can be seen from Fig. 8(a) and 9(a) that the stability time of deformation is mainly concentrated in the low value area. $70.5 \%$ of the vault settlement and $72.9 \%$ of the peripheral convergence can be stable within 30 days, and the sections that are stable for more than 60 days account for a small proportion. The average stability time of vault settlement and convergence is 27.3 days and 25.3 days, respectively. It can be seen from Fig. 8(b) and 9(b) that the distribution of stability time of surrounding rock deformation between highway fault tunnels and railway fault tunnels in 0-20 days is very close. The distribution of stability time of highway fault tunnels in 20-30 days accounts for about $1 / 3$ of the total, which is far greater that that of railway fault tunnels, while the proportion of railway tunnels stability time in high value area is higher. This means that the surrounding rock deformation of highway fault tunnels could enter into stability earlier than railway fault tunnels.

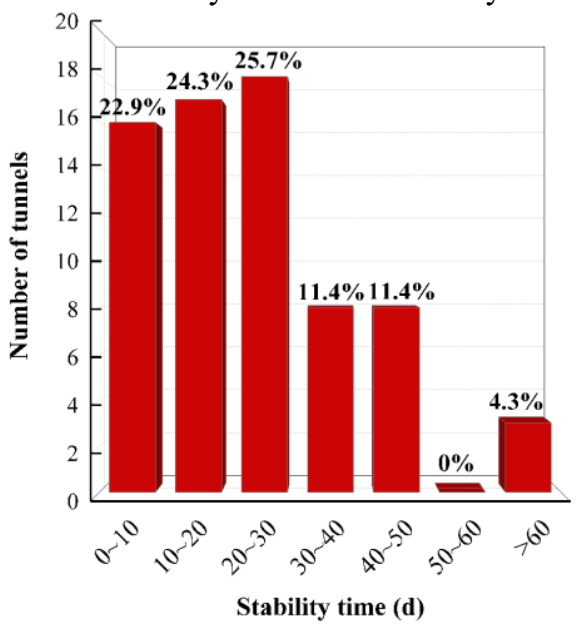

(a)

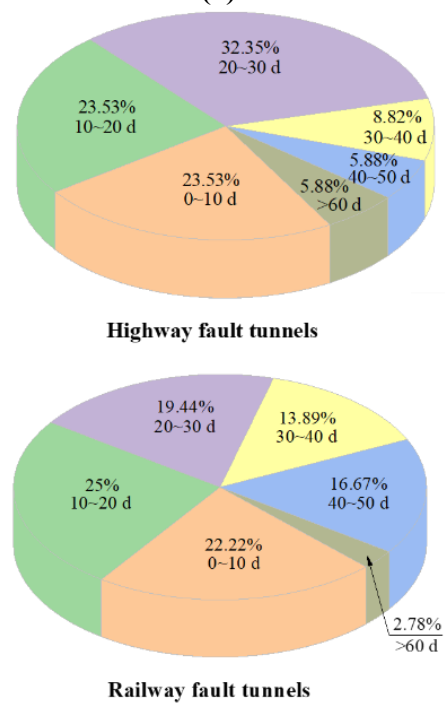

(b)

Fig. 9 The stability time of convergence of fault tunnels

\section{Conclusions}

(1) The overall characteristics of the surrounding rock deformation of the fault tunnels are that the deformation of the railway fault tunnels is larger than that of the highway tunnels and much larger than that of subway tunnels, and the surrounding convergence is greater than the vault subsidence. The problem of excessive deformation of the surrounding rock is common when tunnels are constructed across fault zones.

(2) The surrounding rock deformation of fault tunnels increases with the increases of buried depth and the deformation of railway fault tunnels is more easily affected by buried depth. The larger deformation values of surrounding rock of railway fault tunnels are mainly concentrated in the range of 0.8-0.85 and 1.1-1.2 heightspan ratios, showing significant concentration.

(3) The surrounding rock deformation in fault tunnels 
show significant time effect after excavation, which can be divided into three stages: rapid growth, slow growth and stabilization. Nearly $80 \%$ of the surrounding rock deformation rapid growth stage ended within 15 days, and $64.2 \%$ of the total vertical settlement and $65.6 \%$ of the total horizontal convergence were completed in this stage. $70 \%$ of the surrounding rock deformation tends to be stable within 30 days.

\section{Acknowledgments}

The authors acknowledge the financial support provided by the Department of Transportation Research Fund of Zhejiang Province (Grant No. 2016019).

\section{References}

1. Kimura F, Okabayashi N, Kawamoto T. (1987) Tunnelling through squeezing rock in two large fault zones of the Enasan Tunnel II. Rock Mechanics and Rock Engineering, 20(3): 151-166.

2. Jeon S, Kim J, Seo Y, Hong C. (2004) Effect of a fault and weak plane on the stability of a tunnel in rock-a scaled model test and numerical analysis. International Journal of Rock Mechanics and Mining Sciences, 41: 658-663.
3. Kun M, Onargan T. (2013) Influence of the fault zone in shallow tunneling: A case study of Izmir Metro Tunnel. Tunnelling and Underground Space Technology, 33: 34-45.

4. Wang Y.C, Jing H.W, Su H.J, Xie J.Y. (2016) Effect of a fault fracture zone on the stability of tunnelsurrounding rock. International Journal of Geomechanics, 17(6): 04016135.

5. Liu X.Z, Su J.W, Wang X.X. (2009) Statistical analysis on time effects and evaluation index of deformation stability to NATM surrounding rock deformation. China Civil Engineering Journal, 2009, 42(11): 115-120.

6. Zhao D.P, Yu Y, Wang M.N, Yang J.M. (2009) Study on the deformation regularity and preset deformations for large section tunnels in loess. Modern Tunnelling Technology, 46(06): 64-69.

7. Fang Q, Su W, Zhang D.L, Yu F.C. (2016) Tunnel deformation characteristics based on on-site monitoring data. Chinese Journal of Rock Mechanics and Engineering, 35(09): 1884-1897.

8. China Communications First Highway Engineering Bureau Co., Ltd. (2009) JTG/T F60-2009 Technical rules for highway tunnel construction. China Communications Press, Beijing. 